This item was submitted to Loughborough's Research Repository by the author.

Items in Figshare are protected by copyright, with all rights reserved, unless otherwise indicated.

\title{
Quantifying the mechanical properties of polymeric tubing and scaffold using atomic force microscopy and nanoindentation
}

\section{PLEASE CITE THE PUBLISHED VERSION}

https://doi.org/10.1002/pen.25085

\section{PUBLISHER}

Wiley @ Society of Plastics Engineers

\section{VERSION}

AM (Accepted Manuscript)

\section{PUBLISHER STATEMENT}

This is the peer reviewed version of the following article: NASEEM, R. ... et al, 2019. Quantifying the mechanical properties of polymeric tubing and scaffold using atomic force microscopy and nanoindentation. Polymer Engineering and Science, 59 (5), pp. 1084-1091, which has been published in final form at https://doi.org/10.1002/pen.25085. This article may be used for non-commercial purposes in accordance with Wiley Terms and Conditions for Use of Self-Archived Versions.

\section{LICENCE}

CC BY-NC-ND 4.0

\section{REPOSITORY RECORD}

Naseem, Raasti, Liguo Zhao, Vadim Silberschmidt, Yang Liu, S.K. Eswaran, and S. Hossainy. 2019. "Quantifying the Mechanical Properties of Polymeric Tubing and Scaffold Using Atomic Force Microscopy and Nanoindentation". figshare. https://hdl.handle.net/2134/36899. 


\title{
Quantifying the Mechanical Properties of Polymeric Tubing and Scaffold using Atomic Force Microscopy and Nanoindentation
}

\author{
R Naseem ${ }^{1}$, LG Zhao ${ }^{1}$, VV Silberschmidt ${ }^{1}$, Y Liu $^{1}$, SK Eswaran $^{2}$, S Hossainy ${ }^{2}$ \\ ${ }^{1}$ Wolfson School of Mechanical, Electrical and Manufacturing Engineering, Loughborough \\ University, Loughborough, LE11 3TU, UK \\ ${ }^{2}$ Abbott Vascular, 3200 Lakeside Drive, Santa Clara, CA 95054, USA
}

\begin{abstract}
Measurement of mechanical parameters of polymeric scaffolds presents a significant challenge due to their intricate shape and small characteristics dimensions of their elements - around $100 \mu \mathrm{m}$. In this study, mechanical properties of polymeric tubing and scaffold, made of biodegradable poly (1-lactic) acid (PLLA), were characterised using atomic force microscopy (AFM) and nanoindentation, complemented with tensile testing. AFM was employed to assess the properties of the tube and scaffold locally, whilst nanoindentation produced results with a dependency on the depth of indentation. As a result, the AFM-measured elastic modulus differs from the nanoindentation data due to a substantial difference in indentation depth between the two methods. With AFM, a modulus between 2 and $2.5 \mathrm{GPa}$ was measured, while a wide range was obtained from nanoindentation on both the tube and scaffold, depending on the indentation scale. Changes in the elastic modulus with in-vitro degradation and ageing were observed over the one-year period. To complement the indentation measurements, tensile testing was used to study the structural behaviour of the tube, demonstrating the yielding, hardening and fracture properties of the material.
\end{abstract}

Keywords: Mechanical property; Polymer tube; Polymer scaffold; Nanoindentation; Atomic force microscopy. 


\section{Introduction}

Stents were first used in the 1980s to treat patients with blocked coronary arteries (e.g., stenosis). The devices helped avoid early constrictive remodelling, stabilize vascular dissections and limit arterial recoil. Currently, permanent drug-eluting stents (DES) made of metallic alloys (e.g. Co-Cr alloy) with a polymeric drug-bearing coating are considered the best-in-class treatment option. However, due to the residual risks of restenosis (vessel re-blockage) and late stent thrombosis associated with the permanent metallic stents [1-3], intensive research is ongoing in order to reduce the occurrence of these adverse effects [4-6]. Recently, stents made of bioresorbable materials were developed to overcome the issues observed for permanent metallic stents. Bioresorbable scaffolds provide transient mechanical support to a vessel wall over a period of over 3 months [7]. These devices are intended for full degradation and resorption by the body's metabolic pathways [4], allowing arterial recovery and hence, provide potential for better long term outcomes [6].

Poly-l-lactic acid (PLLA) is widely used in biomedical applications due to its high biocompatibility, moderate degradation rate and pliable physical/chemical nature [8]. PLLA has a high tensile strength and adequate elongation, making it ideal for load-bearing applications [9]. Bioresorbable scaffolds have been investigated since the 1980s, when the first PLLA- based stent was developed by Clark et al. at Duke University [10]. After this, an array of bioresorbable scaffolds were developed including the Igaki-Tamai (Igaki Medical Planning Company, Japan), DESolve (Elixir Medical, CA), Fantom (REVA Medical, CA) and ABSORB BVS (Bioresorbable Vascular Scaffold, Abbott Vascular, CA). The ABSORB BVS was the first scaffold on the market approved by both CE and FDA [11]. The radial strength of ABSORB BVS was comparable to that of common metallic stents, despite the polymer material having weaker bulk mechanical properties than metallic materials. Also, manufacturing manipulations such as polymer-chain alignment can be used to increase the circumferential orientation of crystallite 
segments in polymeric tubes, allowing for optimization of the resistance to radial compression [4].

Testing of bioresorbable polymeric materials used in scaffolds can be carried out in various forms, from assessing the fibre strands, which construct the scaffold, to the bulk material used to extrude the tubing from which scaffolds are laser-cut. Polymeric scaffolds typically undergo multiple processes to achieve the final state. So, there are various factors, which can alter mechanical properties of the material, including processing conditions, material crystallinity and molecular orientation $[9,12,13]$. Leenslag et al. [12] assessed PLLA fibres after a process of hot drawing and dry spinning. With this method, it was found possible to attain high-molecularweight PLLA fibres with a high elastic modulus $(11.4 \mathrm{GPa})$ in comparison to a lower elastic modulus that PLLA typically presents. Weir et al. [9] investigated the effect of processing and sterilization on the molecular weight and crystallinity of a polymer, two factors which can influence the degradation. Tensile testing was carried out on standard dog-bone shaped samples. The findings indicated that at the beginning of the processing method (compression moulding/extrusion), the crystallinity of the polymer decreased pertaining to a low Young's modulus; however, annealing and sterilisation did contribute to an increase in crystallinity along with a modulus increase (618 to $668 \mathrm{MPa}$ for compression-moulded PLLA). Grabow et al. [13] assessed mechanical properties of laser-cut PLLA micro-specimens produced by solution-cast, especially the effects of $\mathrm{CO}_{2}$-laser machining and sterilization methods. Specifically, microdogbone samples with dimension close to the strut size of stents were tested. The results were consistent with those found by Weir et al. [9], confirming that processing and sterilization should not be over-looked in the selection of the stenting material (showing a modulus of $2.9 \mathrm{GPa}$ ), while the effect of laser cutting was found to be less significant. As discussed above, a large variation is reported for the modulus of PLLA (0.6 - $11.4 \mathrm{GPa})$, depending on the processing methods $[9,12,13]$. 
Current ASTM guidelines for testing bioabsorbable stents (F3036-13) are mostly related to general sampling, conditioning, three-point bending, elastic recoil and measurement of securement of stents onto a delivery system [14]. Also, the literature generally highlights the use of standard dog-bone samples or fibres for testing of polymer properties, with limited data on local mechanical properties. Given that polymeric scaffolds undergo processing as part of tailoring their mechanical properties, key insights could be gained by understanding local mechanical properties. To produce ABSORB scaffolds, bulk amorphous PLLA is first extruded into a tube followed by blow moulding, allowing for a $35-55 \%$ increase in crystallinity. This is the result of molecular alignment of polymeric chains in the circumferential direction, which enhances mechanical properties. Then, laser cutting is employed to produce the scaffolds out of the extruded tube according to a specific geometrical design.

In this paper, the main focus is on the local properties of PLLA tube and scaffold in their manufactured states as compared to the macroscopic properties typically reported in the literature. This study was complemented by tensile testing of PLLA tube to study the bulk material response. The effect of one-year in-vitro degradation on local mechanical properties was also investigated to provide additional insights into the performance of bioresorbable polymeric scaffolds.

\section{Experiments}

\subsection{Material and samples}

The samples supplied for this study were poly-lactic acid (PLLA) expanded tubes (Fig. 1a) and lased scaffolds (Fig. 1b) from Abbott Vascular (California, USA). The wall thickness for both the tube and the scaffold is $\sim 150 \mu \mathrm{m}$ and the strut width was $\sim 200 \mu \mathrm{m}$; these dimensions were 
further confirmed with optical imaging (SmartScope Flash 200) and scanning electron microscopy (SEM) after being sputter-coated with gold (Fig. 1c).

\subsection{Atomic Force Microscopy}

Atomic force microscopy (AFM) indentations were carried out using an AFM Explorer (Veeco), with indents made on three locations across the scaffold, i.e., a junction, a link and a U-crest (Fig. 2). Twenty indents were made at each location randomly using a silicon tip with a radius of $10 \mathrm{~nm}$. A $10 \mathrm{nN}$ load was set, and the corresponding displacement was recorded during loading and unloading. A $200 \mathrm{~nm}$ offset was applied to counteract an effect of any possible contaminated residual layer presented on the sample surface. Indents were made on surfaces normal to both the radial and longitudinal axes to assess variability in indenting for these two directions. Using a method proposed by Tang et al. [15], the elastic modulus of the material was calculated on the basis of Sneddon's elastic contact solution by:

$$
\begin{aligned}
& E_{r}=\frac{\propto}{K / A-1} \\
& \frac{1}{E_{r}}=\frac{\left(1-v^{2}\right)}{E}+\frac{\left(1-v_{i}^{2}\right)}{E_{i}},
\end{aligned}
$$

where $E_{r}$ is the reduced modulus of the material related to the contact between the tip and the sample, E and $\nu$ are elastic modulus and Poisson's ratio of the material, $E_{i}$ and $v_{i}$ are the elastic modulus and the Poisson's ratio of the indenter tip (137 GPa and 0.17, respectively [15]). The two parameters $\alpha$ and $A$ define the cantilever-tip property and cantilever sensitivity, respectively. They were obtained through carrying out AFM tests on two reference materials with the known moduli. Here, poly (methyl methacrylate) (PMMA) and polystyrene (PS) were considered with a modulus of $2.76 \mathrm{GPa}$ and $3.55 \mathrm{GPa}$, respectively. Their modulus values were obtained using dynamic mechanical analysis (DMA). The parameter $K$ was defined as the gradient of the 
unloading curve obtained with AFM. Once these values are known, they can be used to quantify the unknown elastic modulus of a material using equation (2). The assumption is made that no plastic deformation of the tip occurs during this calibration procedure [15].

\subsection{Nanoindentation}

Indentation was performed using a Platform 3 indenter (Micromaterials, Wrexham). This was carried out on a nanoscale using a Berkovich tip. A loading rate of $0.5 \mathrm{mN} / \mathrm{s}$ and an unloading rate of $5 \mathrm{mN} / \mathrm{s}$ were chosen, with a 40 -second dwell time at the level of maximum loading. To allow for correction of indentation data with regard to thermal drift, a hold phase of $60 \mathrm{~s}$ was implemented at $80 \%$ of unloading. The elastic modulus of the materials was determined using the Oliver-Pharr theory, based on the slope of the unloading curve (top portion; 20\%) [16]. Using equation (2), the elastic modulus of the material can be calculated with the knowledge of the reduced modulus. Here, the values of $E_{i}$ and $v_{i}$ were $1140 \mathrm{GPa}$ and 0.07 for a diamond indenter tip [17].

\subsection{Tensile test}

Tensile testing of the PLLA tubing was conducted using a 3343 Instron tester, with a displacement rate of $0.042 \mathrm{~mm} / \mathrm{s}$. Two metal inserts were placed into both ends of the tubing and then clamped in the grips (Fig. 3). Five samples were subjected to tensile testing under the same experimental conditions. Here, the schematic drawings in Fig. 3 are to demonstrate that the ASTM standard [18] has been followed in the conduction of tube testing.

\subsection{Effect of degradation}

To assess the lased scaffold's degradation with time, 4-ring sections were sliced from the structure and placed into glass vials filled with phosphate buffer solution (PBS) with a maintained $\mathrm{pH}$ of $7.4 \pm 0.2$, and then incubated at constant temperature $\left(37^{\circ} \mathrm{C}\right)$ to mimic the $\mathrm{in}^{-}$ 
vivo conditions. It is noted that the in-vitro tests here were to ascertain the benchtop degradation profile of the lased scaffold and do not represent the behaviour of the finished good scaffold, which includes additional manufacturing processes such as crimp and sterilization. Over the course of one-year incubation, the PBS was changed every 2 weeks to maintain the right $\mathrm{pH}$ value. Indentation tests (nanoindentation and $A F M$ ) were carried out with the same protocol as previously discussed. Additionally, aged samples (samples stored at room temperature for 365 days; in sealed but not air-tight containers and exposed to light conditions) were also examined to assess the effect of environmental factors on the sample mechanical properties. It is important to note that the mechanical properties of aged samples are not representative of finished good product since the manufacturing process controls/durations are different.

\section{Results and discussions}

\subsection{AFM experiments}

\subsubsection{Tests on PLLA tube}

Average unloading curves for 5 locations tested across the tubing surface with AFM are presented in Fig. 4a. Each location consisted of 4 indents made in a $20 \times 20 \mu \mathrm{m}^{2}$ square area. The unloading curves are acquiescent with one another, suggesting similar material behaviour across the five different regions. To determine the Young's Modulus of the material, the theory proposed by Tang et al. [15] was followed and the gradient of the top portion of the unloading curve was used to calculate the modulus. The average of 20 indents produced a modulus of 2.28 $\mathrm{GPa}(\mathrm{SD}=0.005 \mathrm{GPa})$, adequately representing that of the material at $200 \mathrm{~nm}$ AFM indentation given the low standard deviation.

\subsubsection{Tests on scaffold ring}

Unloading indentation curves for the scaffold ring are shown in Fig. 4b. Five indents were made at 3 different locations, i.e., link, junction and u-crest struts, and their averages were then plotted. 
Again, an overlap of the unloading curves for these locations of the scaffold appear to suggest that, on a nanoscale, mechanical properties of the surface layers of the scaffold appear to be the same across the scaffold. Table 1 details the elastic modulus calculated using the same method as that for the tubing. The values obtained closely align with each other, with low standard deviations, suggesting similar material behaviour up to the depth indented using AFM throughout the scaffold. To further validate this point, indents were also made along the longitudinal axis of the ring to compare with the indents on the outer ring (i.e., radial indents). Values of 2.27 and $2.15 \mathrm{GPa}$ were obtained for the modulus with AFM along the radial and longitudinal directions, respectively. These results again are similar, showing that AFM appeared to give homogenous results with low anisotropy for local elastic response of the material.

In literature, AFM has been used to examine the morphology, and also measure the modulus, of PLLA nanofibres [19-21], and the modulus results showed large variations (from 0.4 GPa to 11 $\mathrm{GPa}$ ) due to different processing techniques used in producing the nanofibres. Consequently, it is very difficult to make direction comparison with the AFM results in literature. In this study, the AFM measurements showed a modulus of $2.28 \mathrm{GPa}$ for tubing and 2.11 2.24 GPa for scaffolds, which are within the range expected for bulk PLLA (2-4 GPa) [22]. Furthermore, our AFM data for both the tubing and ring samples yielded similar elastic moduli, with a range between $2.11 \mathrm{GPa}$ and $2.28 \mathrm{GPa}$, indicating that the surface layers of the polymer structure were almost unaffected during the manufacturing process (i.e., from tubing to scaffold).

\subsection{Nanoindentation experiments}

\subsubsection{Tests on PLLA tube}

An initial load of $10 \mathrm{mN}$ was used to indent specimens to assess their material response at this load. Spacing between the neighbouring indents was set at $30 \mu \mathrm{m}$ to avoid interactions. The load-displacement curves obtained for the polymeric tubing samples are illustrated in Fig. 5a-5c. 
The dwell period can be observed, with the $10 \mathrm{mN}$ load held for 40 seconds at the peak of the loading curve. An increase of displacement occurred during the dwell period, reflecting timedependent (e.g. creep) deformation of the material. A pop-out feature on the unloading curve reflected the hold time at $80 \%$ of unloading for thermal-drift correction, as a result of timedependent creep behaviour. The curve displays elastic and plastic behaviour, apparent from a loop hysteresis and residual imprints on the sample surface made with the Berkovich indenter. Power-law fitting was employed for the unloading curve, for which the Oliver-Pharr methodology was used to calculate mechanical properties. The reduced modulus is the output data, which is then converted into the Young's modulus using Eq. (2). This was calculated as 3.23 $\mathrm{GPa}$ for the tubing materials, which is higher than the value obtained with AFM. Importantly, compared to the AFM results, the Young's modulus in nanoindentation was obtained for much larger depths, i.e., from 1000 to $3000 \mathrm{~nm}$.

Furthermore, an array of indentations was also carried out on the sample, which consisted of a series of single nanoindentation test at a gradually increasing load level between 10 and $25 \mathrm{mN}$. This is apparent (Fig. 5b) in the increasing indentation size and imprints left on the tubing surface. It can be observed that hysteresis and plastic deformation in those indents made at a higher load were larger. The data obtained in the tests is presented in Table 2, showing the increase of maximum indentation and residual depths for each subsequent indent due to the progressive growth of the applied load level. It is also observed from this data set that as the load increased, the Young's modulus declines. A $23 \mathrm{mN}$ load test gave a maximum indentation depth of $2502 \mathrm{~nm}$ and the Young's modulus of $2.5 \mathrm{GPa}$, which is within the expected range for PLLA (2-4 GPa) [22].

Consecutively, 55 indents were made in 2 rows on the surface of the tubing using a load level of $23 \mathrm{mN}$ (Fig. 5c). All the obtained indentation curves exhibited the same trend as that in the 
previous tests. An average modulus of $2.44 \mathrm{GPa}$ was found for the 55 indentation points carried out at $23 \mathrm{mN}$. In comparison to the initial load of $10 \mathrm{mN}$ used, for this 2.3 -fold increase in the load level, a $42 \%$ increase in the maximum depth (1462 versus $2523 \mathrm{~nm}$ ) and $24 \%$ decrease in the modulus (3.7 versus $2.8 \mathrm{GPa}$ ) were found.

As illustrated in Fig. 6, elastic modulus decreased continuously with the increase in the indentation depth and applied load. This decline in Young's Modulus appears to be more rapid for the shallower depths, compared to that for the deeper indentation at higher loads. This could be attributed to an indentation size effect, a phenomena generally observed in polymers and metals, i.e., an increase in hardness and a decrease in plasticity with reduced indentation depth [23].

\subsubsection{Tests on scaffold ring}

Five rings taken from the scaffold samples also underwent the indentation tests. The testing conditions applied to tubing (see Section 3.2.1) were used here, with the same experimental input. Two types of sample were tested - aged and unaged. As previously noted, the mechanical properties of aged samples are not representative of finished good product since the manufacturing process controls/duration are different. The aged data was acquired in order to assess how aliphatic polymer performance changes with time. Load-displacement curves for unaged scaffold rings are given in Fig. 7a. As illustrated by Fig. 7c, a range of depths and moduli were found for indentations at a load of $10 \mathrm{mN}$. Almost a $7 \mathrm{GPa}$ difference can be seen between the largest and the smallest modulus values obtained from indentations carried out on these rings.

Aged scaffold samples exhibited behaviour different from unaged ones. Firstly, their forcedisplacement curves were different (Fig. 7b) from those typically obtained in previous tests on 
unaged samples (Fig. 7a). The aged samples appear to display a more elastic behaviour, with less plastic deformation and enhanced recovery, in comparison to those seen for the unaged sample. Secondly, indentation appeared to peak at around $1500 \mathrm{~nm}$ under $10 \mathrm{mN}$ load for unaged samples, while a depth of $10,000 \mathrm{~nm}$ was reached on the aged samples relating to an elasticmodulus value of $0.06 \mathrm{GPa}$ only. These results are comparable to those seen in both tubing and unaged sample, with the aged sample showing a larger indentation depth and lower elastic modulus. A vast difference was established for the elastic modulus values obtained for two sample sets (Fig. 7c). For the unaged samples, modulus varied between 2 and $9 \mathrm{GPa}$. This range was reduced and remained within $1 \mathrm{GPa}$ for the aged samples tested under the same experimental conditions. Indentation depths for the aged samples were up to 4 times larger than those for the unaged ones.

Semi-crystalline polymers are known to have spatial non-uniformity as a result of the crystalline regions in the material. These areas are scattered randomly and, therefore, it is not possible to assess specific composition of a volume being indented, i.e., amorphous, crystalline or spherulite regions. This could potentially be a reason for the wide range of elastic modulus obtained from nanoindentation, since contribution of amorphous regions could result in lower stiffness while the higher modulus might be associated with the crystalline laminae. Another factor, which could impact on results in polymers, is presence of spherulites which are larger spherical regions formed when the lamellae coagulate, and indentation at these regions would show a high modulus. Also, experimental setup could contribute to the variation in elastic modulus, such as the mounting of the scaffold rings due to their small nature (as opposed to the larger tube samples) as well as the inherence curvature from the tubing. Generally, nanoindentation, as well as AFM, is a method to assess the material properties of local areas (albeit larger than those in AFM), and the results obtained reflect the variability of mechanical properties at such scales associated with semi-crystalline microstructure as well as physical ageing effects. 


\subsection{Tensile experiments}

Tensile experiments were planned to overcome the limitations of AFM and nanoindentation, related to their characterisation of rather small volumes of the polymer. As it was not possible to machine a standard sample such as a dog-bone type sample, the tube was tested as it was. The testing procedure was in close alignment with the ASTM guidelines for testing polymeric samples [18]. According to them, metal plugs are essential to ensure patency of the tube when clamped in the grips. A response of 5 tubing segments to tension is presented in Fig. 8. There is a slight difference for each segment, which may be due to the material anisotropy, but generally the yield point of the samples appears at approximately $110 \mathrm{~N}$, corresponding to a yield stress of 0.071 GPa. Yield stress $(\sigma y)$ was calculated by dividing the force by cross sectional area at the observed yield point. Four samples ruptured at an extension of around $1.5 \mathrm{~mm}$ (strain of 0.03 ), while one sample continually elongated until rupture at $27 \mathrm{~mm}$ extension.

\subsection{Effect of one-year degradation on properties}

The AFM and nanoindentation techniques used to test the virgin-state samples were also employed to assess the effect of one-year in vitro degradation on mechanical property change. With AFM, as seen in Table 3, the effect of degradation on Young's modulus was insignificant. In contrast, a substantial difference was found with nanoindentation for the sample at one-year degradation in comparison to the virgin sample (Fig. 9). A drastic increase in the maximum displacements reached at a smaller load was observed which, in turn, relays to the decreased Young's modulus. At a load of $0.5 \mathrm{mN}$, corresponding to a displacement of $401 \mathrm{~nm}$, a modulus value of $1.47 \mathrm{GPa}$ was obtained and at an increased load of $23 \mathrm{mN}$, the displacement increased proportionally to $17,446 \mathrm{~nm}$ with a modulus value of $0.04 \mathrm{GPa}$. These results, in comparison to the virgin-state data, suggest the occurrence of degradation within the sample at a local level. 
The trend observed in the virgin samples was also noticeable in the degraded ones; an increase in the maximum indentation depth corresponded to a concurrent drop in the modulus. This could be due to the indentation size effect or local modifications of physical properties in the polymer associated with material processing. Ageing and degradation increases malleability of the polymeric chains, leading to a reduction in modulus values as reported here. It could be said based on the obtained data that the effect of ageing is very similar to that of in-vitro degradation at 12 months, with comparable trend lines and modulus ranges. Due to a limited number of indents made on the sample consisting of 2 rings only, some disparity and randomness was introduced to the nanoindentation results. The results presented here are related to local material volumes of lased scaffolds and do not represent the overall bulk material properties of a finished good scaffold. Previously, evaluation of finished good mechanical performance (radial strength) of BVS has indicated qualitative changes in the load response without a reduction in strength at 12 months degradation [24]. Taken together, these results indicate that even with changes in local mechanical properties due to degradation, the bulk mechanical strength can still be maintained.

\section{Conclusions}

This work assessed mechanical properties of bioresorbable polymeric tube and scaffold using the AFM and nanoindentation methods, which provide data for localized volumes, with those in AFM being extremely small. A change of material properties with the indenting depth was observed with nanoindentation, which is a result of various factors such as size effect as well as the non-homogenous microstructure of the semi-crystalline polymer material. In AFM-based studies, the material properties appear consistent across the surface of the samples, due to a very shallow penetration depth. Although both AFM and nanoindentation are indentation techniques, the results presented here are not directly comparable mostly because of the difference in responding volume. The work presented here does illustrate a method to assess local mechanical 
properties of materials, particularly for samples with small dimensions; however, it should be understood that the results are related to local structure property relationship and need an elaborate multi-scale analysis before these data can be correlated to overall bulk material properties of the scaffold.

\section{Acknowledgement}

This work is funded by the British Heart Foundation $\mathrm{PhD}$ research project (Grant number: FS/15/21/31424; Title: Towards controlling the mechanical performance of polymeric bioresorbable vascular scaffold during biodegradation).

\section{Ethical statement}

The research does not involve human and animals experiments.

\section{Conflict of interests}

The authors declare that there is no conflict of interests

\section{References}

1. T Simard, B Hibbert, FD Ramirez, M Froeschl, Y-X Chen, and ER O’Brien, The evolution of coronary stents: A brief review. Can. J. Cardiol., 3035 (2014).

2. H Hara, M Nakamura, JC Palmaz, and RS Schwartz, Role of stent design and coatings on restenosis and thrombosis. Adv. Drug Deliv. Rev., 58377 (2006).

3. ER O’ Brien, X Ma, T Simard, A Pourdjabbar, and B Hibbert, Pathogenesis of neointima formation following vascular injury. Cardiovasc. Hematol. Disord. Drug Targets, 11, 30 (2011).

4. JP Oberhauser, S Hossainy, and RJ Rapoza, Design principles and performance of bioresorbable polymeric coronary scaffolds. EuroIntervention, 5 F15 (2009).

5. CV Bourantas, Y Onuma, V Farooq, Y Zhang, HM Garcia-Garcia, and PW Serruys, 
Bioresorbable scaffolds: Current knowledge, potentialities and limitations experienced during their first clinical applications. Int. J. Cardiol., 16711 (2013).

6. PW Serruys, MJ Kutryk, and AT Ong, Coronary artery stents. N. Engl. J. Med., 354483 (2006).

7. Q Luo, X Liu, Z Li, C Huang, W Zhang, J Meng, Z Chang, and Z Hua, Degradation model of bioabsorbable cardiovascular stents. PLoS One, 9 e110278 (2014).

8. I Grizzi, H Garreau, S Li, and M Vert, Hydrolytic degradation of devices based on poly(dl-lactic acid) size-dependence. Biomater., 16305 (1995).

9. NA Weir, FJ Buchanan, JF Orr, DF Farrar, and A Boyd, Processing, annealing and sterilisation of poly-l-lactide. Biomater., 253939 (2004).

10. RS Stack, RM Califf, HR Phillips, DB Pryor, PJ Quigley, RP Bauman, JE Tcheng, and JC Greenfield Jr, Interventional cardiac catheterization at Duke Medical Center. Am.J. Cardiol., 62 3F (1988).

11. FDA. Absorb GT1 Bioresorbable Vascular Scaffold (BVS) System. (2016). Available at: http://www.fda.gov/MedicalDevices/ProductsandMedicalProcedures/DeviceApprovals andClearances/Recently-ApprovedDevices/ucm509951.htm.

12. JW Leenslag, S Gogolewski, and AJ Pennings, Resorbable materials of poly(L-lactide). V. Influence of secondary structure on the mechanical properties and hydrolyzability of poly(L-lactide) fibers produced by a dry-spinning method. J. Appl. Polym. Sci., 292829 (1984).

13. N Grabow, M Schlun, K Sternberg, N Hakansson, S Kramer, and KP Schmitz, Mechanical properties of laser cut poly(L-Lactide) micro-specimens: Implications for stent design, manufacture, and sterilization. J. Biomech. Eng., 12725 (2005).

14. ASTM. Standard Guide for Testing Absorbable Stents. (2013). doi:10.1520/F3036-13

15. B Tang, AH Ngan, and JB Pethica, A method to quantitatively measure the elastic modulus of materials in nanometer scale using atomic force microscopy. Nanotechnol., 19 
495713 (2008).

16. WC Oliver, GM Pharr, An improved technique for determining hardness and elastic modulus using load and displacement sensing indentation experiments. J. Mater. Res., 7 1564 (1992).

17. E Ozcivici, S Ferreri, Y-X Qin, and S Judex, Determination of bone's mechanical matrix properties by nanoindentation. In: Westendorf J.J. (eds) Osteoporosis. Methods In Molecular Biology ${ }^{\text {TM }}$, Humana Press, 455, 323 (2008).

18. ASTM International. Standard Test Method for Tensile Properties of Plastics. D638-14 (2014). doi:10.1520/D0638-14

19. EPS Tan, and CT Lim, Nanoindentation study of nanofibers. Appl. Phys. Lett. 87123106 (2005).

20. C-C Liao, C-C Wang, and C-Y Chen, Stretching-induced crystallinity and orientation of polylactic acid nanofibers with improved mechanical properties using an electrically charged rotating viscoelastic jet. Polymer (Guildf). 524303 (2011).

21. Q Iqbal, P Bernstein, Y Zhu, J Rahamim, P Cebe, and C Staii, Quantitative analysis of mechanical and electrostatic properties of poly(lactic) acid fibers and poly(lactic) acidcarbon nanotube composites using atomic force microscopy. Nanotechnology, 26 $105702(2015)$.

22. HY Ang, H Bulluck, P Wong, SS Venkatraman, Y Huang, and N Foin, Bioresorbable stents: Current and upcoming bioresorbable technologies. Int. J. Cardiol., 228931 (2017).

23. YV Milman, AA Golubenko, and SN Dub, Indentation size effect in nanohardness. Acta Mater., 597480 (2011).

24. M.B. Kossuth, L.E.L. Perkins, and R.J. Rapoza, Design Principles of Bioresorbable Polymeric Scaffolds. Interv. Cardiol. Clin., 5349 (2017). 


\section{Figure captions}

Figure 1. (a) PLLA tubing; (b) Image of scaffold; (c) SEM image of scaffold.

Figure 2. Images and schematic of areas of interest across a scaffold.

Figure 3. Set up and schematic of tensile test of PLLA tubing.

Figure 4. Force-displacement curves; (a) 5 locations across tubing surface; (b) 3 locations across scaffold (Each curve is an average of 20 indents)

Figure 5. Load-displacement curves for indents made in tube with different peak loads: (a) 10 $\mathrm{mN}$; (b) $10-25 \mathrm{mN}$; (c) $23 \mathrm{mN}$ (imprints shown in inset)

Figure 6. Variation of Young's Modulus with load level and maximum depth of penetration into tube.

Figure 7. Nanoindentation at $10 \mathrm{mN}$ : (a) indentation curves for unaged samples; (b) indentation curves for aged samples; (c) comparison of elastic modulus.

Figure 8. Tensile testing results for PLLA tubing.

Figure 9. Modulus measured with nanoindentation for virgin, aged (12 months) and degraded (one year) samples 


\section{Table captions}

Table 1. Averaged elastic modulus for each location across scaffold.

Table 2. Data for indents on tube made at load levels between 10 and $25 \mathrm{mN}$.

Table 3. Modulus measured with AFM for samples degraded over one-year period. 

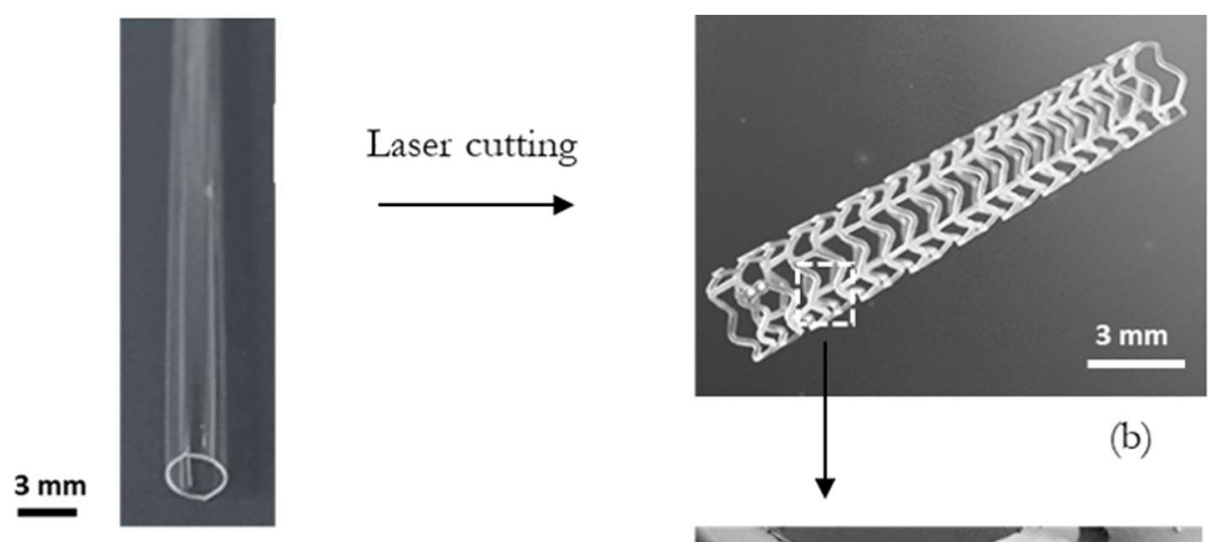

(a)

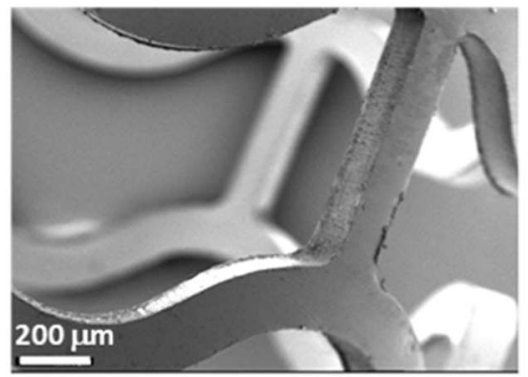

(c)

Figure 1. (a) PLLA tubing; (b) Image of scaffold; (c) SEM image of scaffold.
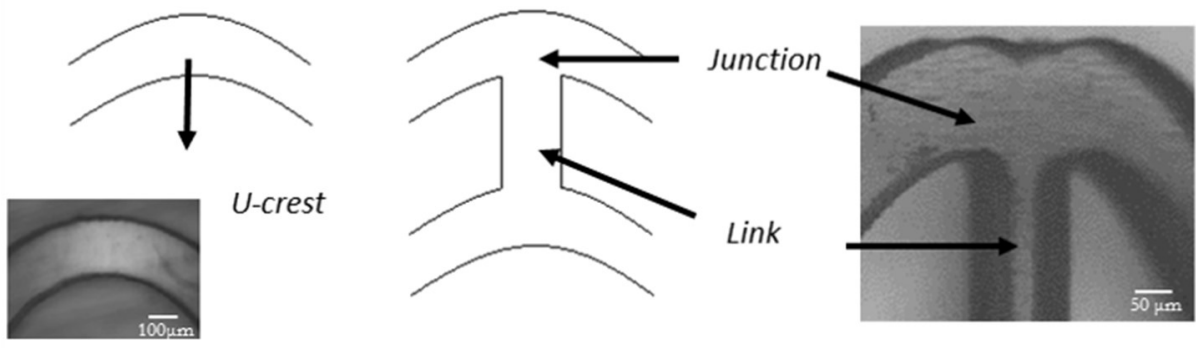

Figure 2. Images and schematic of areas of interest across a scaffold. 

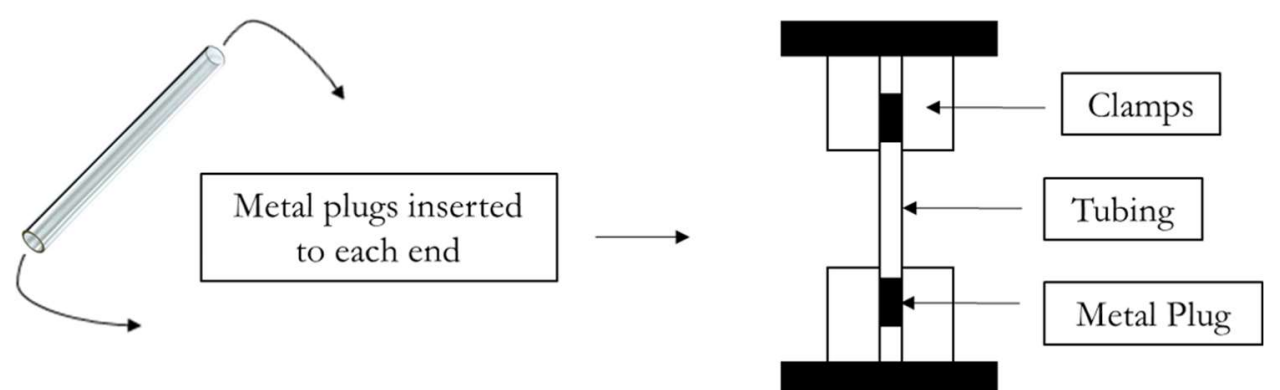

Figure 3. Set up and schematic of tensile test of PLLA tubing.
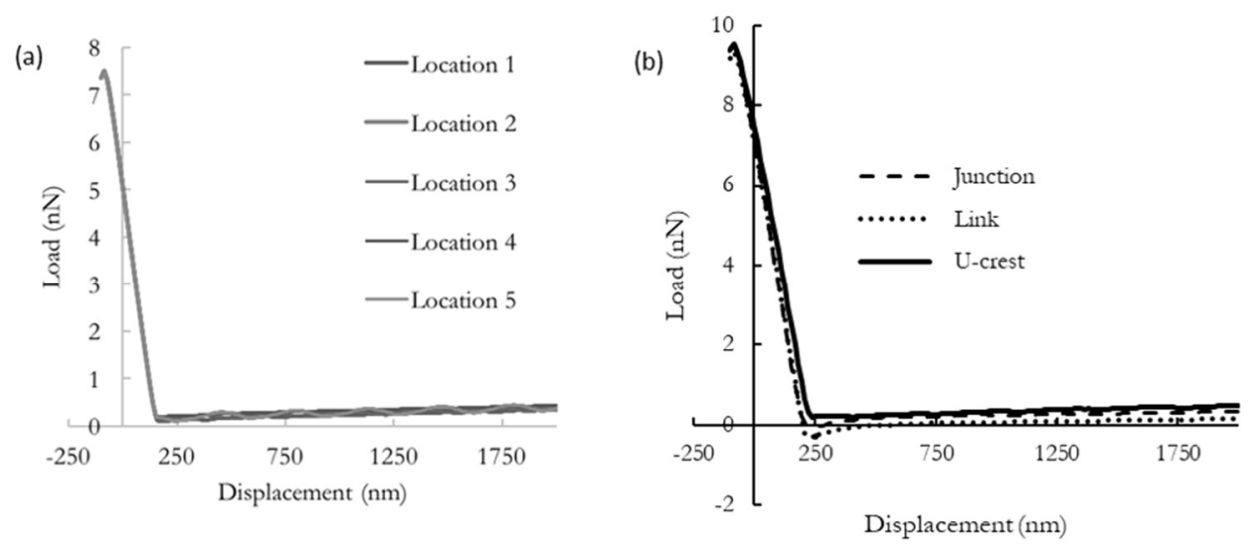

Figure 4. Force-displacement curves; (a) 5 locations across tubing surface; (b) 3 locations across scaffold (Each curve is an average of 20 indents) 
(a)

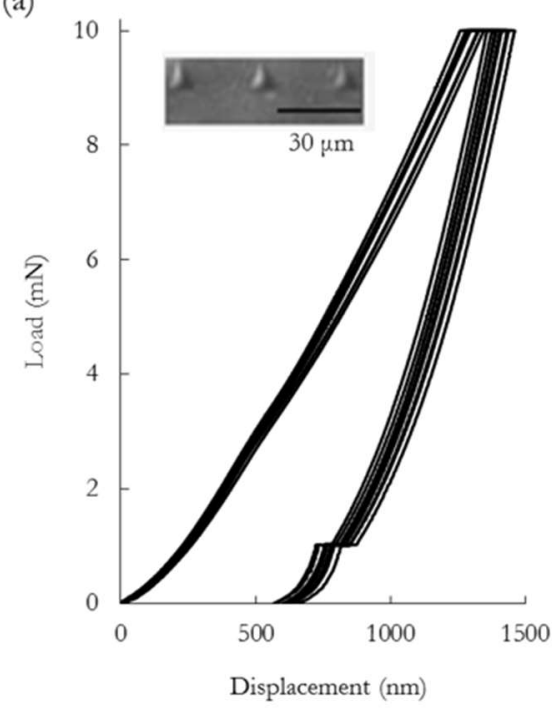

(b)

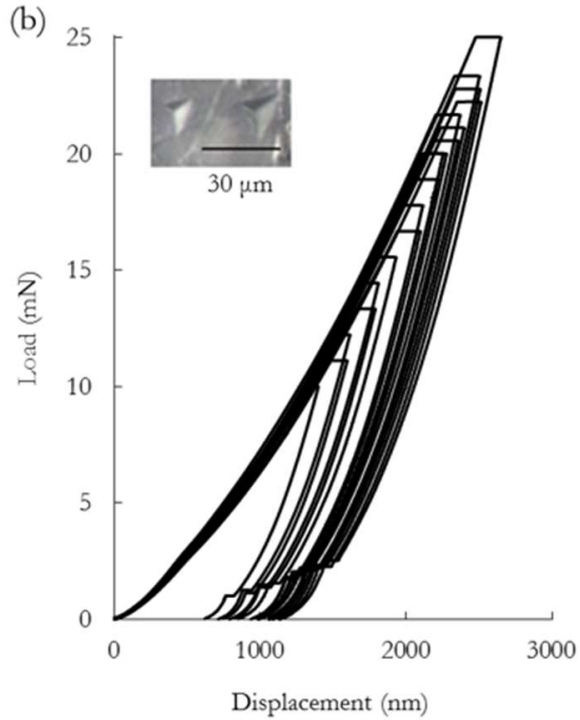

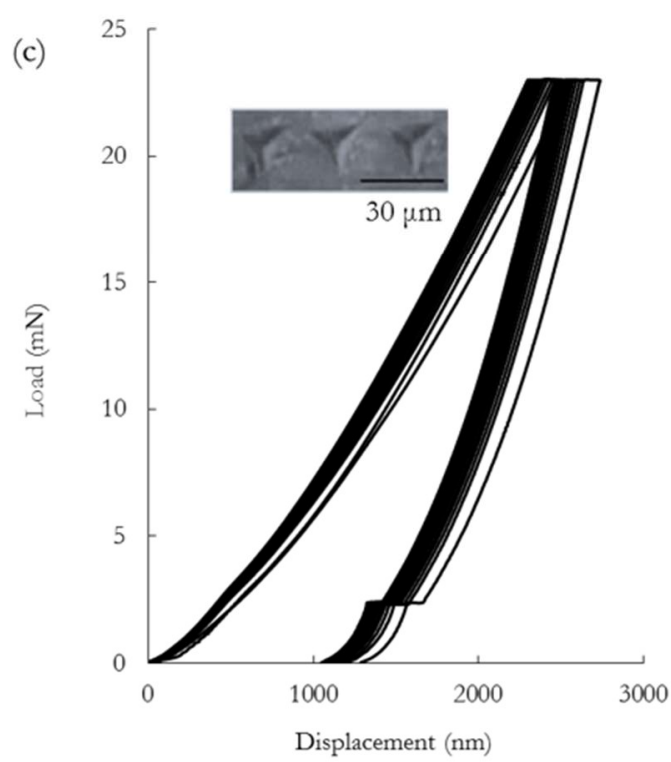

Figure 5. Load-displacement curves for indents made in tube with different peak loads: (a) 10 $\mathrm{mN}$; (b) $10-25 \mathrm{mN}$; (c) $23 \mathrm{mN}$ (imprints shown in inset) 


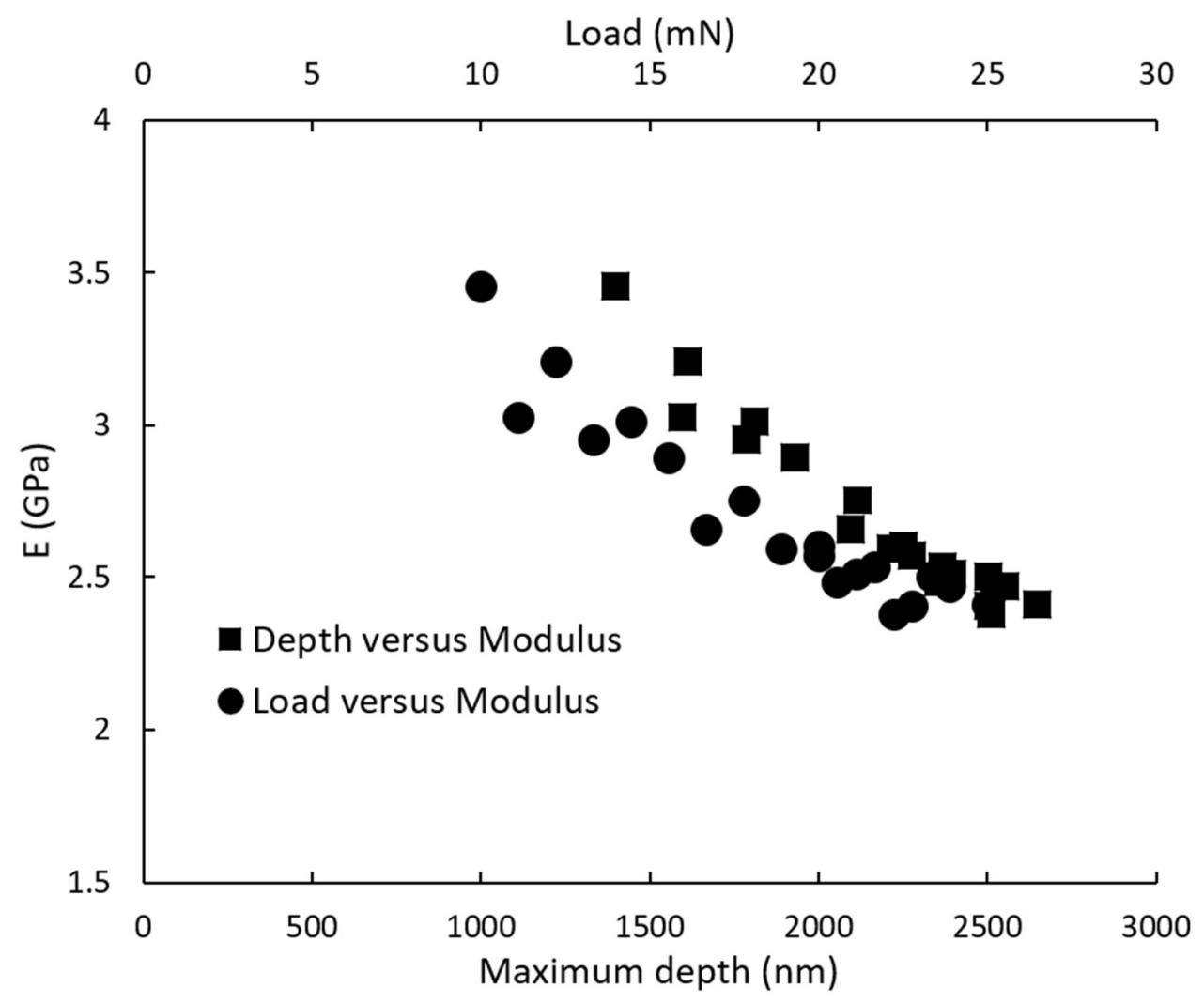

Figure 6. Variation of Young's Modulus with load level and maximum depth of penetration into tube. 

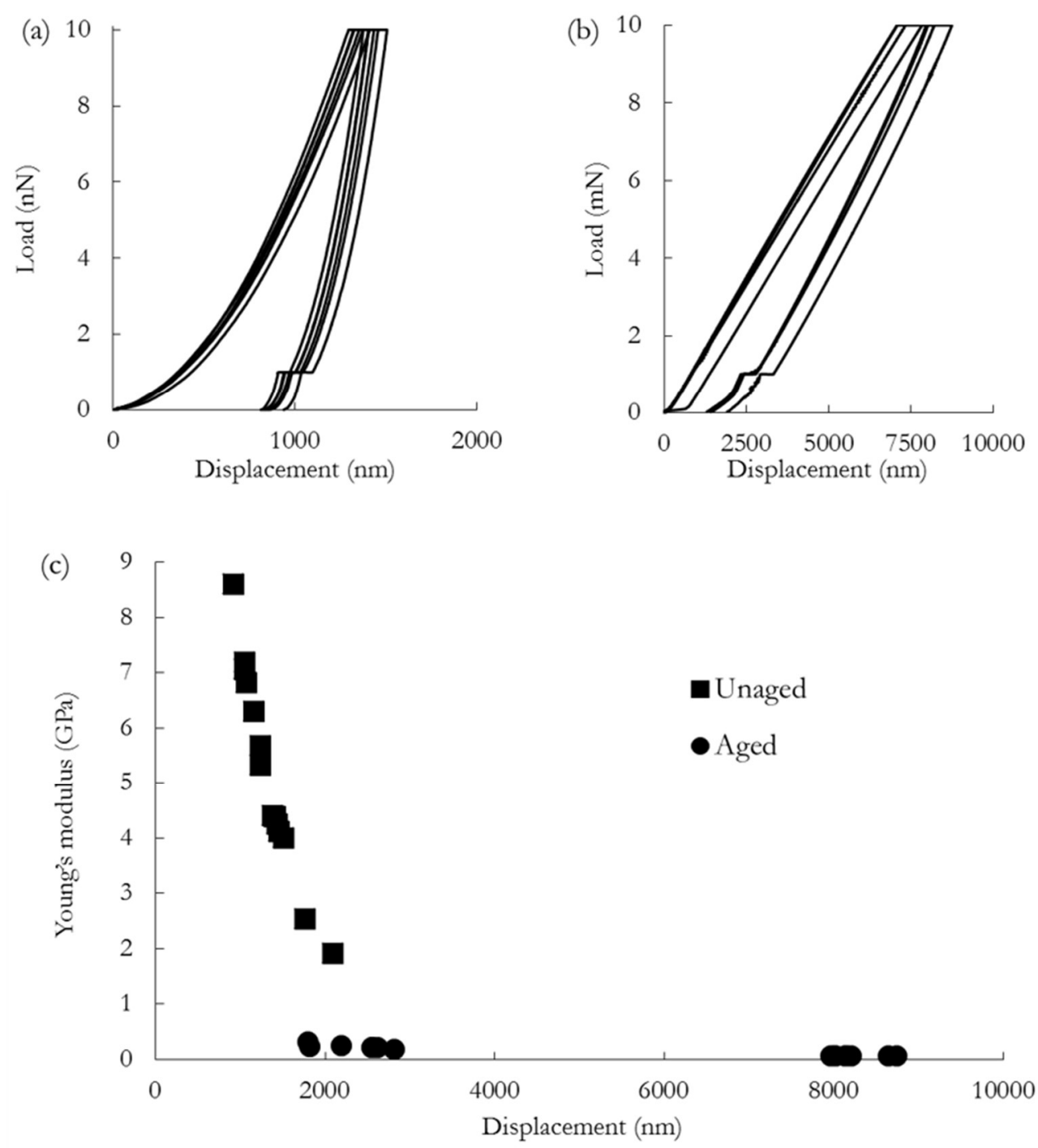

Figure 7. Nanoindentation at $10 \mathrm{mN}$ : (a) indentation curves for unaged samples; (b) indentation curves for aged samples; (c) comparison of elastic modulus. 


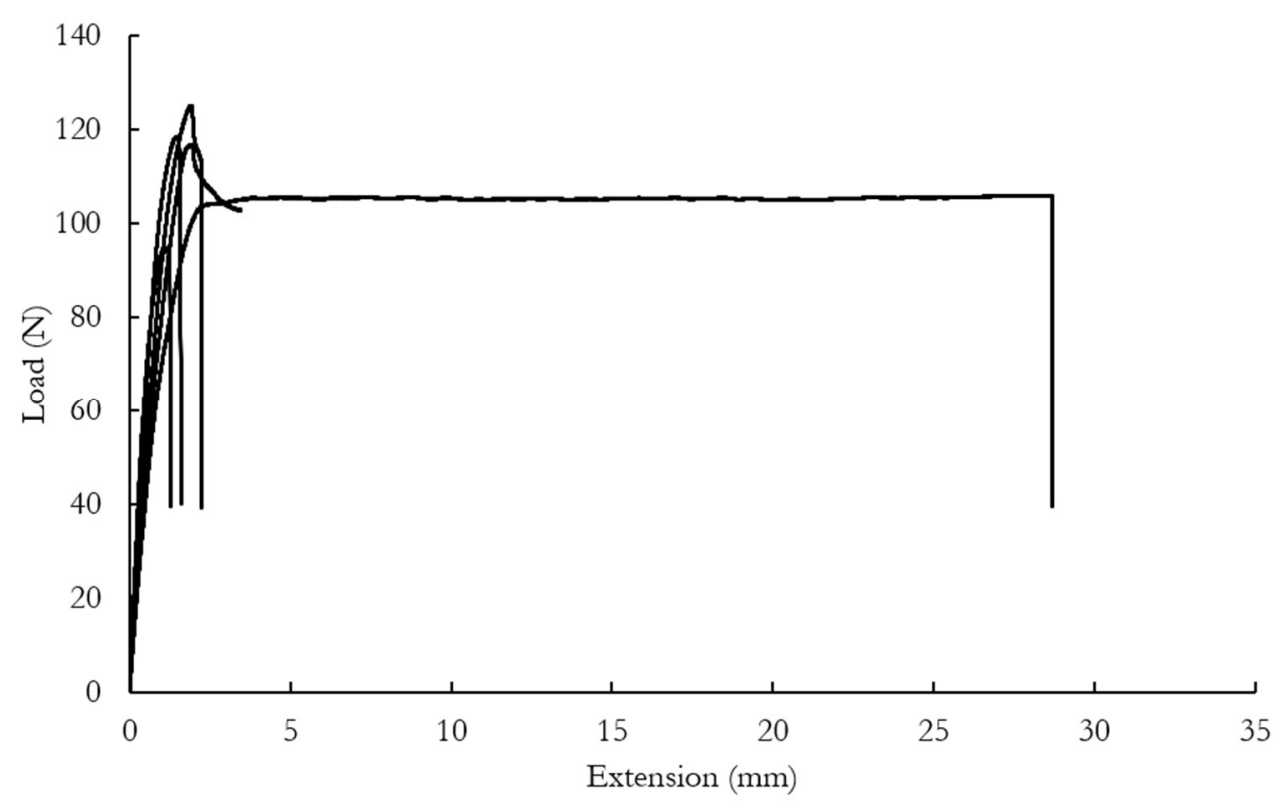

Figure 8. Tensile testing results for PLLA tubing.

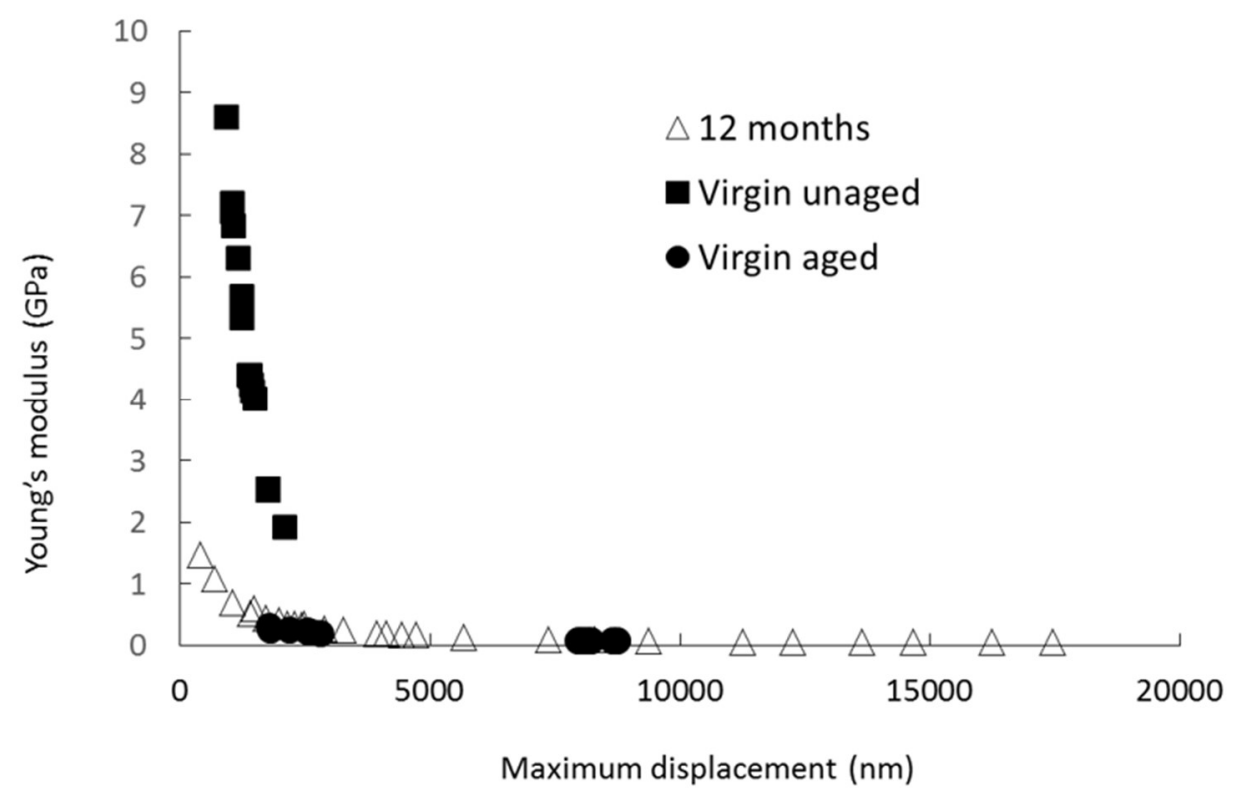

Figure 9. Modulus measured with nanoindentation for virgin, aged (12 months) and degraded (one year) samples. 
Table 1. Averaged elastic modulus for each location across scaffold.

\begin{tabular}{|c|c|c|}
\hline Location & Elastic modulus $(\mathrm{GPa})$ & Standard deviation $(\mathrm{GPa})$ \\
\hline Link & 2.11 & 0.08 \\
\hline U-crest & 2.14 & 0.04 \\
\hline Junction & 2.23 & 0.12 \\
\hline
\end{tabular}


Table 2. Data for indents of tube made at load levels between 10 and $25 \mathrm{mN}$.

\begin{tabular}{|c|c|c|c|c|c|c|}
\hline $\begin{array}{l}\text { Indent } \\
\text { Number }\end{array}$ & $\begin{array}{l}\text { Max. } \\
\text { Depth } \\
(\mathrm{nm})\end{array}$ & $\begin{array}{l}\text { Plastic } \\
\text { Depth } \\
(\mathrm{nm})\end{array}$ & $\begin{array}{l}\text { Max. Load } \\
(\mathrm{mN})\end{array}$ & $\begin{array}{l}\text { Hardness } \\
\quad(\mathrm{GPa})\end{array}$ & $\operatorname{Er}(\mathrm{GPa})$ & $\mathrm{E}(\mathrm{GPa})$ \\
\hline 1 & 1397.25 & 1084.58 & 10.00 & 0.35 & 3.95 & 3.45 \\
\hline 2 & 1595.43 & 1251.41 & 11.11 & 0.29 & 3.47 & 3.03 \\
\hline 3 & 1614.45 & 1259.84 & 12.22 & 0.31 & 3.67 & 3.21 \\
\hline 4 & 1787.54 & 1413.22 & 13.33 & 0.27 & 3.38 & 2.95 \\
\hline 5 & 1809.24 & 1410.79 & 14.44 & 0.30 & 3.45 & 3.01 \\
\hline 6 & 1929.40 & 1512.72 & 15.56 & 0.28 & 3.31 & 2.89 \\
\hline 7 & 2096.65 & 1651.90 & 16.67 & 0.25 & 3.05 & 2.66 \\
\hline 8 & 2113.78 & 1656.44 & 17.78 & 0.26 & 3.15 & 2.75 \\
\hline 9 & 2214.92 & 1717.84 & 18.89 & 0.26 & 2.97 & 2.59 \\
\hline 10 & 2251.94 & 1731.23 & 20.00 & 0.27 & 2.98 & 2.60 \\
\hline 11 & 2275.40 & 1756.46 & 20.00 & 0.26 & 2.95 & 2.57 \\
\hline 12 & 2356.12 & 1824.12 & 20.56 & 0.25 & 2.84 & 2.48 \\
\hline 13 & 2396.72 & 1869.41 & 21.11 & 0.25 & 2.88 & 2.51 \\
\hline 14 & 2367.88 & 1816.27 & 21.67 & 0.27 & 2.90 & 2.53 \\
\hline 15 & 2511.38 & 1949.42 & 22.22 & 0.24 & 2.72 & 2.38 \\
\hline 16 & 2504.63 & 1930.56 & 22.78 & 0.25 & 2.76 & 2.41 \\
\hline 17 & 2502.01 & 1938.73 & 23.33 & 0.25 & 2.87 & 2.50 \\
\hline 18 & 2550.43 & 1977.28 & 23.89 & 0.25 & 2.83 & 2.47 \\
\hline 19 & 2646.58 & 2054.68 & 25.00 & 0.24 & 2.76 & 2.41 \\
\hline
\end{tabular}


Table 3. Modulus measured with AFM for samples degraded over one-year period.

\begin{tabular}{|c|c|c|}
\hline Area & $\begin{array}{c}\text { Young's } \\
\text { Modulus (GPa) }\end{array}$ & $\begin{array}{c}\text { Standard } \\
\text { Deviation } \\
(\mathrm{GPa})\end{array}$ \\
\hline Link & 2.09 & 0.02 \\
\hline U-crest & 2.07 & 0.028 \\
\hline Junction & 2.09 & 0.015 \\
\hline
\end{tabular}

